\title{
GRAVITATION AND JET INDUCED VELOCITIES IN THE \\ NARROW LINE REGION OF ACTIVE GALAXIES
}

\author{
MARK WHITTLE \\ Astronomy Department \\ University of Virginia \\ Charlottesville VA 22903 \\ U.S.A.
}

\section{INTRODUCTION}

The basic question I want to address is : "What mechanism or mechanisms accelerate the gas in the narrow line region of active galaxies, yielding the observed profile shapes." At the present time there is no generally accepted answer to this question. Part of the problem dates back to the first few Seyferts that were discovered which, in retrospect, seem to have had anomalously broad lines. For example NGC 1068, MKN 3, and MKN 78 have [OIII] $\lambda 5007 \mathrm{FWHM} \sim 1000 \mathrm{~km} \mathrm{~s}^{-1}$, which is clearly greater than anything associated with a normal galaxy velocity fields. An obvious implication was that these high velocities were in some way related to the activity. This view was reinforced by the discovery of a correlation between [OIII] FWHM and non-thermal radio luminosity (Wilson and Willis 1981). When many radio sources were found to have linear double or triple morphology (e.g. Ulvestad and Wilson 1984a,b), a natural explanation seemed to be outflowing radio jets which stir up the narrow line region and thus generate the correlation between line width and radio luminosity.

On the other hand, some facts seemed to undermine this "active" acceleration picture. First, the [OIII] FWHM do not correlate with the overall level of activity. For example, the mean FWHM for Seyfert 2s, Seyfert 1s and QSOs are the same, despite an enormous range of non-thermal luminosity (Whittle 1985b). Second, as more [OIII] line widths were measured, it became clear that the mean FWHM is only $\sim 350 \mathrm{~km} \mathrm{~s}^{-1}$, much less than previously thought. Such a velocity is similar to a typical stellar velocity in a spiral bulge of $M_{B} \sim-21.5$. Although this seems large, it is in fact typical of Seyferts, which tend to inhabit relatively massive early type spirals. Thus, we have a second possibility that for the majority of Seyferts, the basic acceleration mechanism may be gravitational (Whittle $1985 \mathrm{a}, \mathrm{b})$.

To test this latter possibility it is important to look for explicit correlations between [OIII] linewidth and diagnostics of the bulge potential. Two such diagnostics are the rotation amplitude of the galaxy and the bulge absolute magnitude.

\section{[OIII] FWHM AND GALAXY ROTATION}

The observed rotation amplitude is simply the peak to peak velocity difference on the flat part of the rotation curve, measured either from long slit optical emission line 

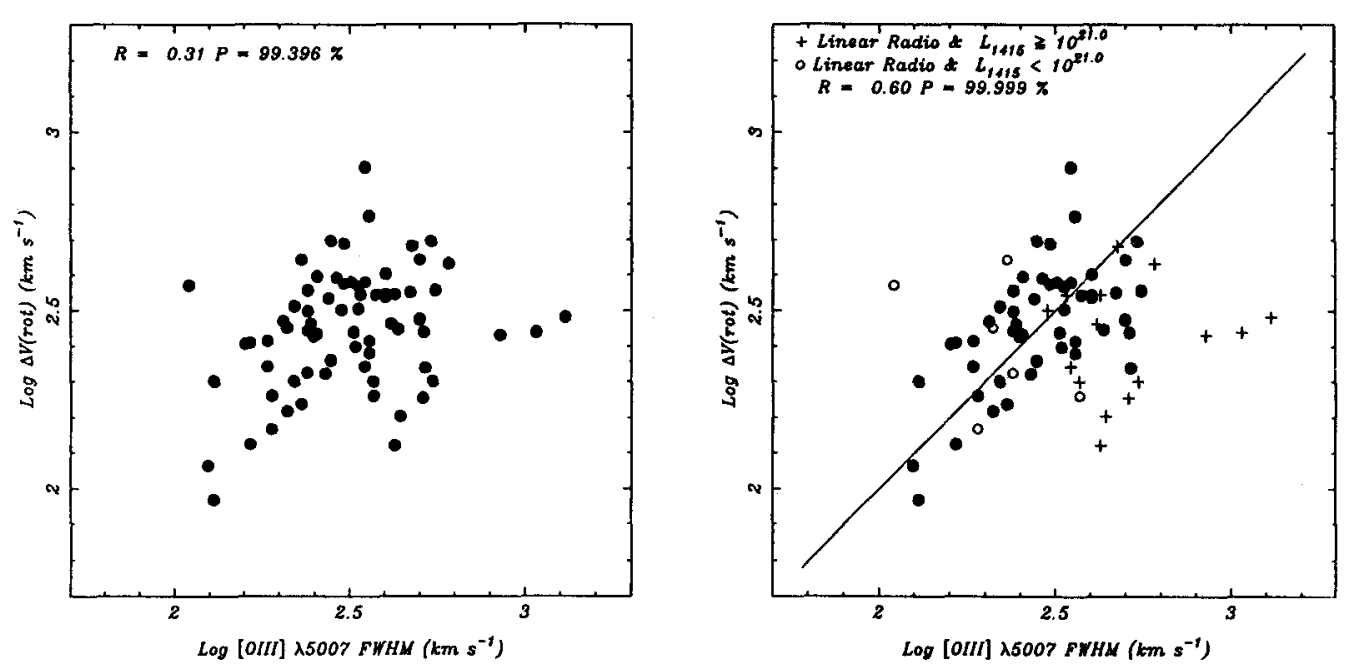

Figure 1 (left) : Plot of [OIII] FWHM against observed galaxy rotation amplitude for about 60 Seyferts.

Figure 2 (right) : Same data as Figure 1, objects flagged by radio properties.

studies or single beam HI profiles. Figure 1 shows a plot of Log [OIII] FWHM against Log observed rotation amplitude, $\Delta \mathrm{V}$, for about 60 Seyfert galaxies. There is a general trend with considerable scatter. The overall correlation strength is only moderate : $99.4 \%$ (two tailed). The diagram becomes much more interesting when the radio properties of the individual objects are considered. Figure 2 shows exactly the same data but those objects which have been classified by Wilson and Ulvestad (1984a,b) as having linear radio morphology on the Kpc scale have been flagged differently. Luminous linear radio sources are plotted as plusses, weaker linear radio sources as open circles, the rest as filled circles. There is a clear tendency for the luminous linear radio sources to have anomalously broad [OIII] lines and contribute most of the high velocity scatter. The rest of the sample falls on a tight correlation with linear correlation coefficient 0.6 and very high significance. The best fit straight line falls almost exactly on the line $y=x$. In other words, the [OIII] FWHM not only correlate with but are equal to the observed rotation amplitude.

We conclude from this that the basic acceleration mechanism is gravitational. For some objects there is an additional acceleration mechanism associated with the linear radio structure, and this almost certainly results from the interaction of bipolar jets with the surrounding medium.

\section{JET INTERACTIONS}

Let us first consider the radio jet interaction briefly, before returning to discuss the gravitational processes. A detailed description of 10 Seyferts with linear radio morphology is given by Whittle et al. 1988 . Figure 3 shows the archetype MKN 78. The spectrograph slit was placed along the radio axis to yield high resolution [OIII] $\lambda 5007$ line profiles running east-west across the nuclear regions. In addition to a rotating component, there is a redshifted component which is physically located close to the eastern radio lobe, and also a 

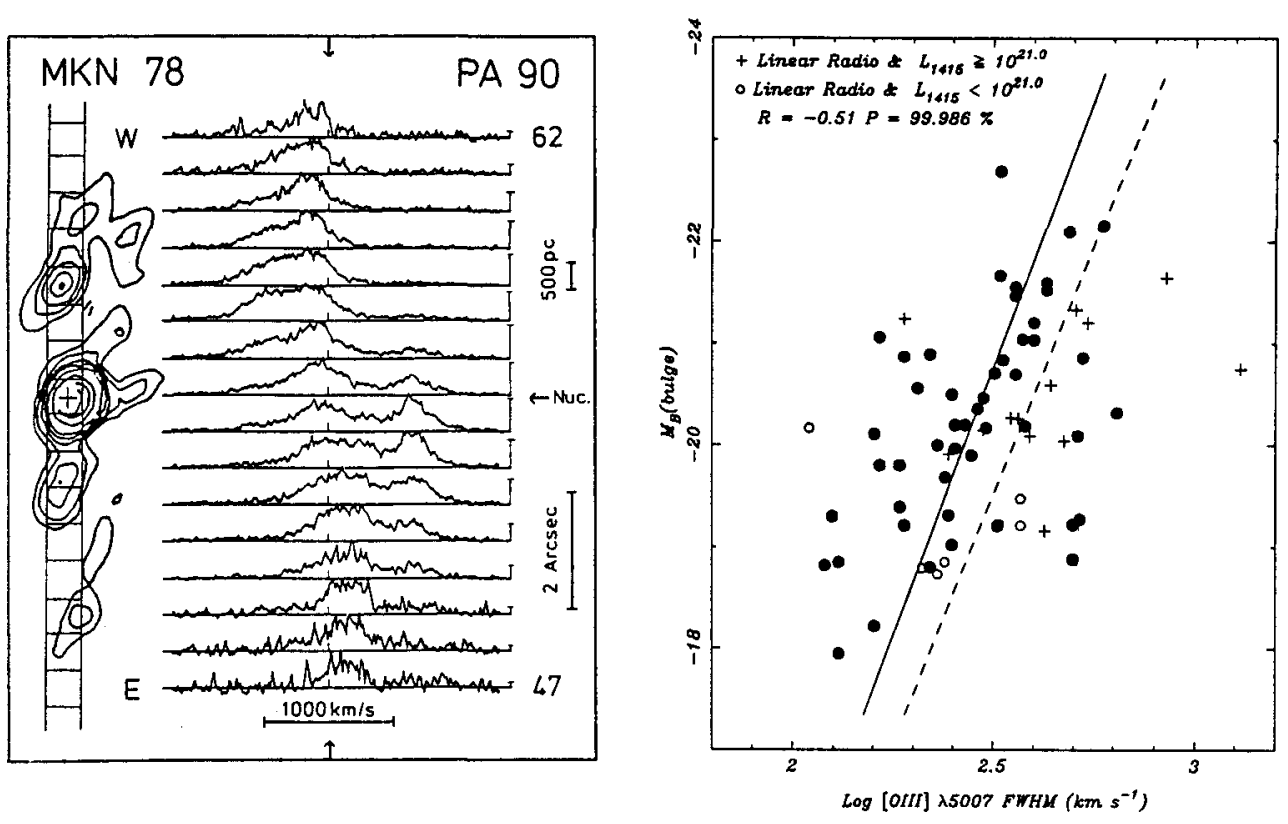

Figure 3 (left) : Long slit [OIII] $\lambda 5007$ observation of MKN 78 (autoscaled) with radio continuum map plotted at the same scale (from Whittle et al 1988).

Figure 4 (right) : Plot of [OIII] FWHM against bulge absolute blue magnitude, with best fit line (solid) and relation between stellar velocities and bulge magnitudes for normal galaxies (dashed line)

blueshifted, somewhat blended, component which is located close to the western radio lobe. The simplest interpretation is that the two high velocity components are accelerated locally by the interaction of a jet with the interstellar medium. It isn't yet clear whether this is an entrainment process or accelaration by an advancing bow shock. Both these processes need to be modelled in more detail.

\section{GRAVITATIONAL VELOCITIES}

Returning now to the group of objects for which gravity seems to be the dominant acceleration mechanism. How can the rotation amplitude, which is measured on large scales in the disk of the galaxy, be related to a highly nuclear velocity measurement taken, typically, with a $2-3$ arcsec aperture? There are two scenarios to account for this, although both are probably unrealistic extremes. First, it might be that the rotation velocity remains high right into the nucleus, so that there is significant rotational smearing even within a small aperture. Observations of normal galaxy rotation curves suggest that this is not the explanation since the nuclear rotation gradients are too shallow. However, some galaxies have recently been found to have rapidly rotating nuclear stellar disks with very steep rotation curves (e.g. Franx and Ilingworth 1988, Jedrzejewski and Schechter 1988). If Seyferts possess such disks and these have rotation amplitudes similar to the disk amplitude, then rotational smearing can indeed contribute to the correlation shown in Figure 2. Second, although the rotation curve may be low within a nuclear aperture, the stellar velocity 
dispersion remains high across the bulge. If the ionised gas moves anything like the stars, then a nuclear aperture will yield a broad profile. Now, Whitmore and Kirshner (1981) find that the central velocity dispersion is proportional to the rotation amplitude with only a weak Hubble type dependence. Hence the correlation between rotation amplitude and [OIII] FWHM. In fact, by using their relation to infer nuclear velocity dispersion from rotation amplitude, it can be shown that [OIII] FWHM is proportional to and approximately equal to the inferred stellar velocity dispersion.

An independent demonstration of this is shown in Figure 4. Log [OIII] FWHM is plotted against bulge absolute magnitude (in $B$ ). This bulge magnitude has been derived from the total absolute galaxy magnitude and a bulge/total ratio inferred from normal spiral galaxies of the same Hubble type. There is a moderately strong correlation with a best fit (solid) line which lies parallel to (and slightly displaced from) the classic $\mathrm{L} \sim \sigma^{4}$ relation between luminosity and stellar velocity dispersion for spheroidal systems (shown as the dashed line).

Thus, there are two independent demonstrations of gravitational origin to the [OIII] FWHM - correlations with rotation amplitude and correlations with bulge absolute magnitude.

\section{ROTATION OR DISPERSION ?}

It may be possible to distinguish between the two extremes described above (pure rotation and pure dispersion) by using the galaxy inclination. There are really two rotation measures, the observed rotation amplitude, $\Delta \mathrm{V}$, and the inclination corrected or true rotation amplitude, $\Delta V_{c}=\Delta \mathrm{V} / \sin i$. If rotation in the plane of the galaxy dominates the velocity field of the NLR then there should be a tighter correlation between [OIII] FWHM and $\Delta V$ than between [OIII] FWHM and $\Delta V_{c}$. Conversely if dispersion velocities dominate in the NLR then there should be a weaker correlation between [OIII] FWHM and $\Delta V$ than between [OIII] FWHM and $\Delta V_{c}$, since [OIII] FWHM would not itself suffer projection effects. Comparing the two correlations for Seyfert galaxies with well measured inclinations in fact shows no significant differences in their correlation strengths. This result is consistent with an intermediate situation in which both rotation and dispersion velocity fields are present. The result also agrees with a direct analysis of the inclination dependence of [OIII] FWHM (Whittle 1985a,b).

\section{[OIII] LINE WINGS}

The previous discussion considers only the [OIII] line core width, as measured by the FWHM parameter. Do similar correlations exist for the base and wings of the [OIII] profile, which are presumably produced by gas residing closer to the nucleus? The correlations with rotation amplitude and bulge magnitude are progressively weaker for base width, FW20, and wing width, IPV20, parameters. Unfortunately, the interpretation of this result is not clearcut. First, the results seem consistent with the plausible notion that nongravitational acceleration processes may become more important closer to the active nucleus itself. While intuitively appealing, the weak point of this explanation is that the base and wing widths do not seem to depend on overall level of activity (i.e. they do not correlate with non-thermal luminosity or Seyfert type). Second, it is possible that the base and wing widths are still determined by the gravitational potential but that close to the nucleus the gravitational velocities are significantly different from the average bulge velocities measured on larger (arcsec) scales. For example, a dense star core possibly (though not necessarily) 
surrounding a black hole could be dynamically quite isolated form the rest of the bulge. Finally, the breakdown in correlation for base and wing width parameters may simply reflect the fact that while these parameters are well defined observationally they are not well defined physically. A particular problem with the wing width parameters is that they are quite sensitive to the intensity ratio of the core and wings. It may be that a component fitting parameterization scheme, similar to that employed by Pelat et al (1981) for example, would recover the correlation strength between wing widths and gravitational diagnostics.

\section{SUMMARY}

The main result of this study has been to show that the [OIII] FWHM in Seyfert galaxies correlates quite tightly with tracers of the bulge gravitational potential, implying that gravity is usually the dominant acceleration mechanism for the ionised gas in the NLR. For objects with luminous linear radio sources, there is usually an additional acceleration mechanism which is almost certainly associated with a jet interacting with the interstellar medium. Somewhat more tentative results come from an analysis of the inclination dependence of these correlations and suggest the NLR velocity field is only partly confined to the plane of the galaxy. Finally, the correlations between base and wing width and gravitational diagnostics are less good than the correlation with core width, although the interpretation of this is ambiguous.

\section{REFERENCES}

Franx, M., and Ilingworth, G.D., 1988, Astrophys. J. Lett., 327, L55.

Jedrzejewski, R.I., and Schechter, P.L., 1988, Astrophys. J. Lett., 330, L87.

Pelat, D., Alloin, D., and Fosbury, R.A.E., 1981, M.N.R.A.S., 195, 787.

Ulvestad, J.S., and Wilson, A.S., 1984a, Astrophys. J., 278, 544.

Ulvestad, J.S., and Wilson, A.S., 1984b, Astrophys. J., 285, 439.

Whitmore, B.C., and Kirshner, R.P., 1981, Astrophys. J., 250, 43.

Whittle, M., 1985a, M.N.R.A.S., 213, 1.

Whittle, M., 1985b, M.N.R.A.S., 213, 33.

Whittle, M., Pedlar, A., Meurs, E.J.A., Unger, S.W., Axon, D.J., and Ward, M.J., 1988 Astrophys. J., 326, 125.

Wilson, A.S., and Willis, A.G., 1981, Astrophys. J., 240, 429. 